\title{
Acquisition and elaboration of superficial three-dimensional images in plastic and reconstructive surgery: Applications
}

\author{
C. Alfano, P. Mezzana, N. Scuderi \\ Department of Plastic and Reconstructive Surgery, University of Rome "La Sapienza", Italy
}

Address for correspondence: Dr. Paolo Mezzana, Via Merulana 61/A, 00185 Rome, Italy. E-mail: pmezzana@yahoo.it

\section{ABSTRACT}

Since 1970, as computed axial tomography machines became easily available and became more sophisticated, image acquisition techniques and analysis improved, developed rapidly and became very useful in medical diagnosis. Today it is possible to examine either the anatomic and functional aspects of deep body organs and tissues including all the minute details as well as their morphological relations with superficial structures. Through precise graphic elaboration programs we can obtain information about spatial relations which was not possible using simply classical clinical examination. It is possible, for example, to "point out" an area of interest from its anatomic context, to carry out virtual cleavage of planes and to measure volumes and distances. New developments in image acquisition systems permit transfer of three-dimensional data directly from existing objects. The three -dimensional computerized axial tomography for deep structures, and the laser scanners for superficial structure, quickly furnish (1-3 seconds for the laser scanners) useful information to plan the surgical operation.

When analysing 3D-imaging techniques it is important to appreciate their utility for the planning and the follow up of surgery, particularly for the head and thorax, where the three dimensional evaluation is better than the classic double dimensional system that is incomplete and difficult to interpret. In the future, these systems will be important for the best aesthetic and functional results and above all for reducing the surgical time.

\section{KEY WORDS}

Surface Images, three dimensions, analysis

\section{INTRODUCTION}

ince 1970, as computed axial tomography machines became easily available and became more sophisticated, image acquisition techniques and analysis improved, developed rapidly and became very useful in medical diagnosis. Today it is possible to examine either the anatomic and functional aspects of deep body organs and tissues including all the minute details as well as their morphological relations with superficial structures. ${ }^{1}$

Through precise graphic elaboration programs we can obtain information about spatial relations which was not possible using simply classical clinical examination. It is possible, for example, to "point out" an area of interest from its anatomic context, to carry out virtual cleavage planes and to measure volumes and distances.

A perfect knowledge of the body district's morphology 
and topography as well as surgical simulation based on the patient's three-dimensional model, allows the surgeon to plan the operation with extreme precision and accuracy, to improve manual dexterity and to test new surgical approaches. ${ }^{1}$

In surgical fields it is important to make a reliable spatial representation of body sections, especially when defining and planning dissection and reconstruction procedures and when correcting morphological anomalies. ${ }^{1}$ This need is particularly felt in serious trauma and in the treatment of congenital and acquired dysmorphologies. In the past correct osteotomy simulation, or planning graft morphology and tissue flaps was not possible on a digital model. Volume and distance spatial measurements allow us to follow-up the results of the performed surgical procedure.

This paper discusses the possibility of making threedimensional models from various materials, based on data collected with digital images.

The indications for this method are congenital craniofacial dysmorphologies, trauma, ${ }^{1}$ implantology, corrective surgery on results of neoplastic resections and inborn deformities. $^{1}$

\section{ACQUISITION OF THREE-DIMENSIONAL IMAGES: THE SCANNER}

New developments in image acquisition systems permit transfer of three-dimensional data directly from existing objects. $^{2}$ The scanner is an instrument that scans real objects of any dimension and represents them in millions of data points that can be processed to create threedimensional models.

The three-dimensional scan devices can be grouped in 3 major categories:

- contact scan methods

- passive scan methods

- active scan methods

Active scanners irradiate the object with a source of light energy and evaluate the modifications in the reflection of this energy due to its interaction with the object surface. Current technologies use different types of light energy. Scanning time depends on the device and the object's dimension. The range is 1.5 to 15 seconds with a resolution of several hundred micrometers. However, because these devices are based on optical scan methods, they are not effective for surfaces that reflect or completely absorb the source of light. ${ }^{3}$

\section{Structured light scanner}

This technique is based on projecting a light pattern on the object and to analyse the interaction of this pattern with the object's surface. A video-camera acquires the image and then a dedicated software reconstructs the three-dimensional profile. ${ }^{4}$ Structured light scanners are used to scan objects to get a three-dimensional image, which can be visualized on a computer screen. The operator can select different points of view on this image, therefore catching details normally hidden in a simple bi-dimensional picture. ${ }^{5}$

\section{Laser scanner}

In this device a laser light scans the object measuring the reflection of the surface elements in the laser visual field..$^{8-10}$

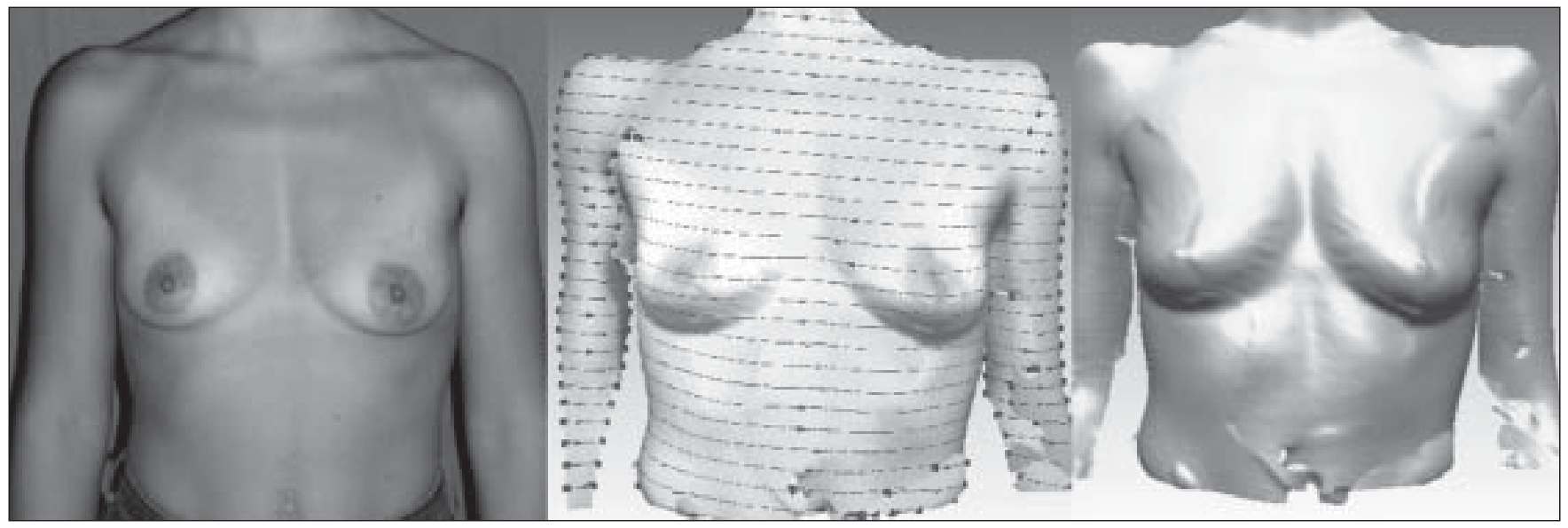

Figure 1: On the left a traditional bidimensional image of breast. On the center and right images tridimensional image of the same patient. In the last two we can appreciate the depression over the nipple areola complex that is not possible with the first image 
This scanner is an effective and rapidly working instrument. Laser three-dimensional scanning allows digitalization of different types of objects and surfaces,

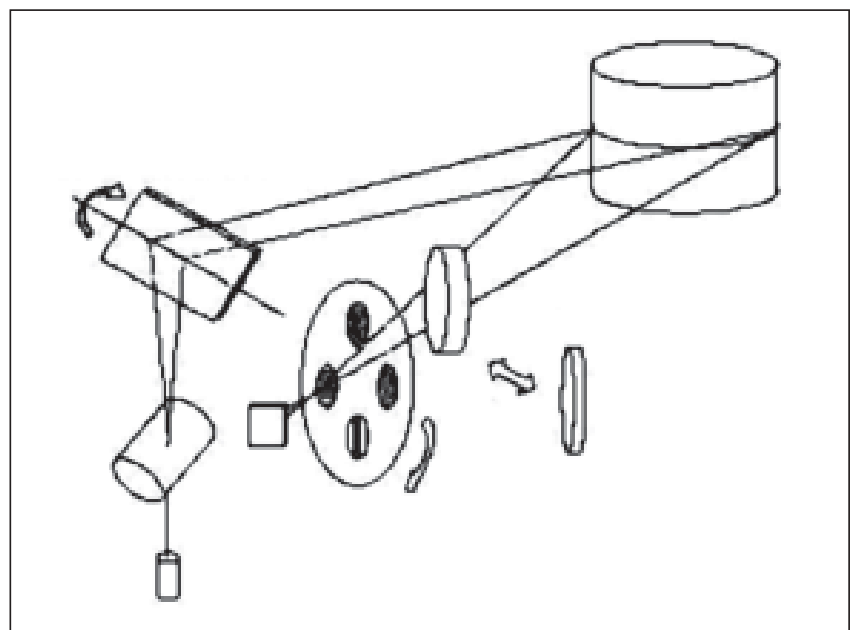

Figure 2: 3D laser scanner in detail, giving accuracy to a fraction of a millimeter. ${ }^{6}$ Images are acquired through the triangulation method. Laser triangulation is a stereoscopic active technique calculating object distance through a directional source of light and a video camera.

The source emits a laser plane of light, whose position is fixed and known. The intersection of this plane with the surface produces a line. Through a video-camera sensitive to laser light frequency (CCD, charge-coupled device), it is possible to observe how this line is modeled on the target, drawing out spatial coordinates of lighted points. ${ }^{9}$ The object moves through a translatory or rotatory movement, by means of a motorized transport system. The luminous line "brushes" over the surface and it is possible to have a complete reconstruction of the object. A similar system works with fixed object and laser plane in movement. The colour is acquired by the passage of

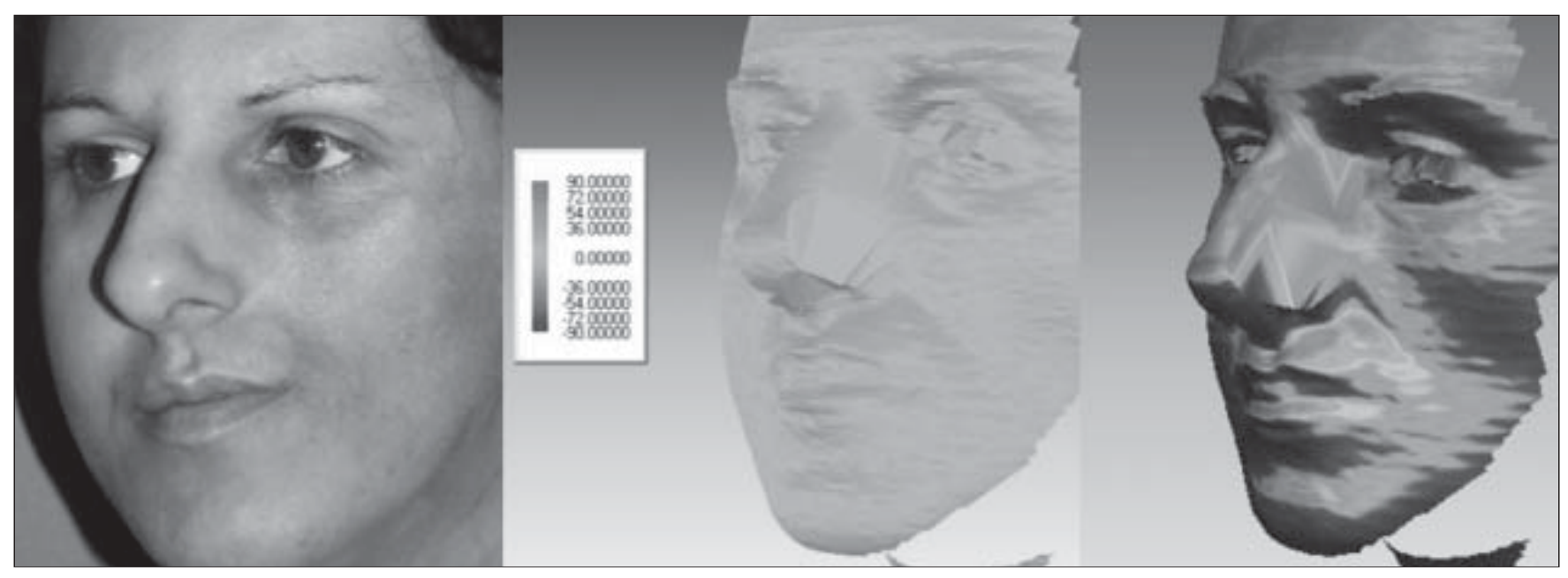

Figure 3: On the left a patient affected by Romberg's disease traditional photo. On the center and right images the tridimensional analysis of the surfaces.

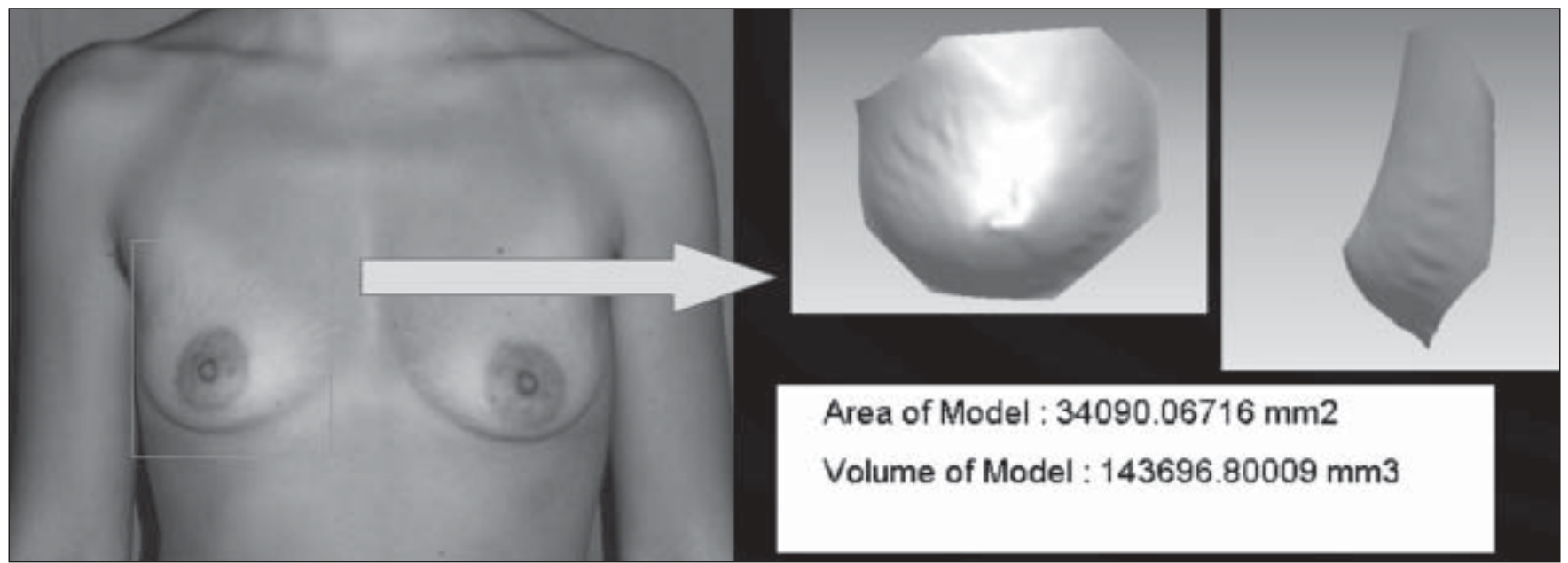

Figure 4: 3D volume measuring of the breast. Preoperative view. 
the rays reflected by the object through four filters positioned inside the CCD (charge-coupled device). The dedicated software then applies post-processing corrections if necessary and finally a the data compiled to create a three-dimensional image that can be exported.

\section{MATERIALS AND METHODS}

The goal of the study was to to test in 15 cases the use of a three-dimensional laser scanner (Minolta VI-700) and appropriate software (Rapid Form, INUS Technology Inc.) in one of the most important Departments of Plastic and Reconstructive Surgery in Italy. In 9 patients a threedimensional volumetric analysis was performed before augmentation mammoplasty, in order to predict the aesthetic result of the operation and the data were compared with what was obtained post-operatively. In 3 patients we measured the degree of breast asymmetry before the augmentation mammoplasty, in order to establish preoperatively the volume difference and choose the appropriate mammary implants to correct the defect. In 3 patients, affected by Romberg's syndrome, we used the system to evaluate the degree of volume improvement of the involved hemi-face after a cycle of polylactic acid infiltrations (Sculptra ${ }^{\circledR}$, Aventis).

\section{RESULTS}

In the 9 patients affected by mammary hypoplasia we demonstrated successfully the usefulness of the presurgical simulation to predict with a good degree of accuracy post-surgical results. We compared the three dimensional simulation images and the three dimensional post-operative images. In the 3 patients affected by breast asymmetry, we demonstrated good aesthetic results. The pre-operative assessment of the three dimensional volume assisted us in choosing the right volume implants which in turn gave better correction of the asymmetry. In the 3 patients affected by Romberg's syndrome we demonstrated that the use of the three dimensional image analysis systems allows objective measurement of the improvement in facial volumes and to compare the symmetry of the two hemi-faces.

\section{DISCUSSION}

The asymmetries of the face are deformities in which the anatomical reference points, the bending, the surfaces and the volumes are irregularly positioned. In the past, two-dimensional techniques and direct measures, such as X-ray scans or photographs were used to plan the surgical intervention. However, they were inadequate to describe the three-dimensional surfaces, particularly of the most irregular zones.

The three-dimensional computerized axial tomographies ${ }^{7}$ for deep structures, and the laser scanners for superficial structure, quickly furnish (1-3 seconds for the laser scanners) useful information to plan the surgical operation. They allow analysis of the distances and the volumes and to simulate the transposition of anatomical units. They are important in the post-operative followup and for the production of three-dimensional solid models that are used in the production of individualised implants. ${ }^{8}$ The three-dimensional scanners are used for the qualitative and quantitative evaluation of the deformities through a correct and rapid "morphometric analysis" of the surfaces. ${ }^{9}$

In our experience with the acquisition of threedimensional models of the face we were able to evaluate objectively the improvement of soft tissue volumes in Romberg's syndrome in 3 patients, by analyzing the concavity and the convexity of the face in a colour map.

The superficial scanner systems are very effective for breast surgery. The breast superficial scanner can measure the mammary gland volumes. This data guarantees the prosthesis implant with a correct volume to fill up the deficit or asymmetry assuring an optimal aesthetic result. Galdino's studies on the technique of three dimensional images acquisition for breast surgery pathology, ${ }^{12}$ also demonstrate the utility of three dimensional systems to obtain the necessary data (volumes, forms, etc) for a correct surgical planning. We used the system in 9 patients to simulate an augmentation mammoplasty before the operation and then we compared the result of the preoperative surgical simulation and the postoperative situation, demonstrating the high predictive capability of the three dimensional analysis.

We used the laser scanner in 3 patients affected by breast asymmetry in order to establish the volume differences, to choose the right volume mammary implant, and demonstrated a good aesthetic result with an almost complete correction of the malformation.

An exhaustive literature search as well as the analysis of the results in our department we feel that the three dimensional superficial image elaboration will have an 
important role in plastic and reconstructive surgery. ${ }^{13,14}$

\section{CONCLUSIONS}

With the three dimensional images obtained from a scanner it is possible to:

1. Simulate a surgical operation: with the visualization of a three dimensional image we can select an area of interest, analyse and modify its morphology. ${ }^{13}$ The surgeon can discuss the expected final result with the patient. ${ }^{14}$

2. Qualitatively and quantitative measures: we can obtain information about superficial balance and distance in minimal time. ${ }^{15}$

3. Produce custom made implants and scaffolds for bioengineered tissues: the association of CAD and CAM permits the production of "made to order" implants and scaffolds as matrix of bioengineered tissues. This is important for better pre-operative planning and for final aesthetic and functional result. It is also important for the reduction of surgical time and for the followup (form variation, ${ }^{15}$ oedema, and inflammatory process etc).

\section{REFERENCES}

1. Udupa JK, Gabor TH, Vannier MW. 3D Imaging in Medicine $3^{\text {rd }}$ Ed. New York: CRC Press LLC; 2000.

2. Tartan Z, Pozzi MF, Pozzi MR. Ottimizzazione dei parametri di scansione e di elaborazione nella ricostruzione 3D in TC del massiccio facciale. La Radiologia Medica 1995;89:578-85.

3. Preda L, La FA, Di MEM, et al. Traumi complessi del massiccio facciale: Apporto diagnostico dell'imaging TC multiplanare e 3D ottenuto con tecnica spirale. La Radiologia Medica 1998;96:17884.

4. Halazonetis DJ. Acquisition of 3-dimensional shapes from images. Am J Orthod Dentofacial Orthop 2001;119:556-60.

5. Raskar R, Fuchs H, Welch G, Lake A, Cutts M. 3D Talking heads: Image based modeling at interactive rates using structured light projection. Computer Science 1998;98:17.

6. Savarese S, Scansione tridimensionale con metodi a luce debolmente strutturata. www.vision.caltech.edu

7. Bhatia G, Vannier MW, Smith KE, Commean PK, Riolo J, Young VL. Quantification of facial surface change using a structured light scanner. Plast Reconstr Surg 1994;94:768.

8. O'Grady KF, Antonyshyn OM. Facial asymmetry: Threedimensional analysis using laser surface scanning. Plast Reconstr Surg 1999;104:928.

9. Pinna V, Marchi MD, Puzzoli A, et al. La TC 3D nella patologia maxillo-facciale. Rivista di Neuroradiologia 1993;6:19-35.

10. Binder WJ, Kaye A. Reconstruction of postraumatic and congenital facial deformities with three-dimensional computerassisted custom-designed implants. Plast Reconstr Surg 1994;94:75.

11. Coward TJ, Watson RM, Scott BJ. Laser scanning for the identification of repeatable landmarks of the ears and face. $\mathrm{Br} \mathrm{J}$ Plast Surg 1997;50:308-14.

12. Galdino GM, Nahabedian M, Chiaramonte M, Geng JZ, Klatsky S, Manson P. Clinical applications of three-dimensional photography in breast surgery. Plast Reconstr Surg 2002;110:5870.

13. Okumura H, Chen LH, Tsutsumi S, Oka M. Three-dimensional virtual imaging of facial skeleton and dental morphologic condition for treatment planning in orthognathic surgery. Am J Orthod Dentofacial Orthop 1999;116:126-31.

14. Xia J, Samman N, Yeung RWK, et al. Computer-assisted threedimensional surgical planning and simulation. 3D soft tissue planning and prediction. Int J Oral Maxillofac Surg 2000;29:2508.

15. Ismail SFH, Moss JP. The three-dimensional effects of orthodondic treatment on the facial soft tissues: A preliminary study. Br Dental J 2001;192:104-8.

\title{
Annoucement
}

The 5th National Conference and International Update of The Indian Society of Cleft Lip, Palate and Craniofacial Anomalies will be held in Guwahati, Assam, India on 23rd to 25th March, 2006. Dr Mukund R. Thatte will be the President.

\author{
For details contact \\ Dr. Seema Rekha \\ Organizing Secretary, \\ 5th ISCLP and CA, 2006. \\ Subansiri Apartment, Govt. Press Rd. \\ Bamunimaidan. \\ Guwahati -781021 \\ Phone No. 0361-2551590 ® \\ $9864012894(\mathrm{~m})$ \\ E-mail seemarekha@hotmail.com \\ Website: www.isclpca2006.org
}

